\title{
Quantitative assessment of fibrosis and steatosis in liver biopsies from patients with chronic hepatitis $\mathrm{C}$
}

\author{
A M Zaitoun, H Al Mardini, S Awad, S Ukabam, S Makadisi, C O Record
}

\begin{abstract}
Backgrounds-Hepatic fibrosis is one of the main consequences of liver disease. Both fibrosis and steatosis may be seen in some patients with chronic hepatitis C and alcoholic liver disease (ALD).

Aims-To quantitate fibrosis and steatosis by stereological and morphometric techniques in patients with chronic hepatitis $\mathrm{C}$ and compare the results with a control group of patients with ALD. In addition, to correlate the quantitative features of fibrosis with the Ishak modified histological score.

Materials and methods-Needle liver biopsies from 86 patients with chronic hepatitis $C$ and from 32 patients with alcoholic liver disease (disease controls) were analysed by stereological and morphometric analyses using the Prodit 5.2 system. Haematoxylin and eosin and Picro-Mallory stained sections were used. The area fractions $\left(A_{A}\right)$ of fibrosis, steatosis, parenchyma, and other structures (bile duct and central vein areas) were assessed by stereological method. The mean diameters of fat globules were determined by morphometric analysis.
\end{abstract}

Department of Histopathology, University Hospital, Queen's Medical Centre, Nottingham NG7 2UH, UK

A M Zaitoun

Department of Medicine, The Royal Victoria Infirmary, Newcastle upon Tyne, UK

H Al Mardini

S Makadisi

C O Record

Department of Medicine, Al Jazeera Hospital, PO Box 2427, UAE

S Awad

Department of Medicine, University of Al AIN, PO Box

15258, UAE

S Ukabam

Correspondence to: Dr Zaitoun

abd.zaitoun@mail.qmcuh-tr. trent.nhs.uk

Accepted for publication 4 December 2000 evaluating hepatic fibrosis and steatosis in chronic hepatitis $C$. They are most useful for assessing the origin, location, and the stage of fibrosis. Stereology and morphometry are recommended for the quantitation of fibrosis and steatosis, particularly for the evaluation of new treatment strategies in patients with chronic hepatitis $\mathrm{C}$.

(F Clin Pathol 2001;54:461-465)

Keywords: alcoholic liver disease; hepatic fibrosis; hepatitis C; morphometry; steatosis; stereology

Acute hepatitis $\mathrm{C}$ develops into chronic hepatitis in more than $50 \%$ of patients. $^{1{ }^{2}} \mathrm{~A}$ proportion of patients with chronic hepatitis $\mathrm{C}$ progress to cirrhosis and hepatocellular carcinoma. ${ }^{3}$ Hepatic fibrosis is one of the most important features of chronic hepatitis C. The accurate assessment of hepatic fibrosis is important to study the natural history and the prognosis of patients with chronic hepatitis C. Fibrosis is also seen in patients with alcoholic liver disease (ALD) ${ }^{4-6}$ and other forms of nonalcoholic fatty liver disease (NAFLD).$^{7-9}$ Steatosis is another morphological feature that can be seen in patients with chronic hepatitis $\mathrm{C},{ }^{10-13}$ ALD, ${ }^{56}$ and NAFLD. ${ }^{7814}$ There are several methods for assessing hepatic fibrosis and the progression of fibrogenesis in clinical practice. Chemical methods are used to measure the concentration of collagen in liver tissue. ${ }^{15-17}$ This is a good method but requires a large sample of liver tissue. Conventional routine histological examination is the most common method, although it is subjective and not quantitative. A histological semiquantitative scoring system for the evaluation of hepatic fibrosis has been described. ${ }^{18-20}$ Morphometric assessment of hepatic fibrosis has been considered the gold standard technique, ${ }^{21}$ and has been used to assess hepatic fibrosis in experimental animals ${ }^{22}$ and in clinical practice. ${ }^{2} 1824$ Fat in liver disease has been assessed by semiquantitative methods ${ }^{14}$ by automated computerised procedures, ${ }^{25}{ }^{26}$ and biochemically in liver biopsy specimens. ${ }^{27}$

Therefore, the accurate assessment of the quantity of fibrosis and steatosis is important for the pathological, clinical, and prognostic evaluation of patients with chronic hepatitis C.

In our present study, we have chosen to focus our attention on patients with chronic hepatitis $C$ versus ALD as a disease control group and have concentrated on the quantification of hepatic fibrosis and steatosis by stereological 
and morphometric techniques. We also compared the quantitative values of fibrosis with the Ishak modified scoring system. ${ }^{19}$

\section{Materials and methods}

One hundred and eighteen liver biopsy specimens were evaluated from patients with chronic hepatitis C (64 UAE, 22 UK) and ALD (32 patients, UK) as disease controls. The mean age of the patients with chronic hepatitis C was 39.5 years (range, 18-65) and 60 were men. The mean age of patients with ALD was 49.8 years (range, 30-69) and 22 were men. In addition to clinical manifestations, all patients with hepatitis $\mathrm{C}$ had positive serological and immunological tests for hepatitis $\mathrm{C}$ (second generation tests). None of these patients had a history of alcohol intake. All patients with alcoholic liver disease had a long history of alcohol intake with clinical and biochemical evidence of chronic liver disease. None of the patients with chronic hepatitis C had received antiviral treatment before the biopsy procedure. Liver biopsy specimens were fixed in $4 \%$ buffered formal saline and embedded in paraffin wax. Sections were cut at $5 \mu \mathrm{m}$ thickness and were stained with haematoxylin and eosin $(\mathrm{H} \& \mathrm{E})$, reticulin, the Picro-Mallory stain, and other special stains. The H\&E stain was used at two levels. All liver biopsies from patients with chronic hepatitis $\mathrm{C}$ were also assessed in relation to the Ishak modified score. ${ }^{19}$ The score of fibrosis was recorded as follows: 0, no fibrosis; 1 , fibrous expansion of some portal tract areas; 2 , fibrous expansion of most portal tract areas; 3, fibrous expansion of portal tract areas with occasional portal-portal bridging; 4, fibrosis with portal-portal and portal-central bridging; 5, pronounced bridging with occasional nodules; and 6, probable or definite cirrhosis.

Steatosis was assessed in H\&E stained sections by morphometric analysis.

\section{STEREOLOGY}

Stereology (area fraction; $A_{A}$ ) is used to obtain quantitative information about the volume, area, length, and numerical density in three dimensional structures using a simple point counting technique or a computer program. In chronic hepatitis $\mathrm{C}$ and ALD, the changes are usually uniform throughout the liver; therefore, biopsy sampling can be considered unbiased for assessing the area fraction. The $\mathrm{A}_{\mathrm{A}}$ of steatosis, unchanged parenchyma, portal tract, central veins, and fibrosis in all zones were assessed by stereological analysis using an objective magnification of $\times 10$ (Nikon microscope). Stereological assessment was carried out using a computerised image analysis system (Prodit 5.2; BMA, Amsterdam, the Netherlands). A monitor screen with parallel Weibel overlay grid with 72 points at a distance of $31.83 \mu \mathrm{m}$ was used to count fat globules, unchanged parenchyma, portal tract areas, central veins, and fibrosis (Picro-Mallory stain). At least 1000 points covering the above features were counted in each liver biopsy specimen and the whole biopsy area was covered at one level of sectioning. At least 13 fields were selected for measurement to include all zones, portal tracts, and vein areas.

\section{MORPHOMETRY}

Morphometry is the measurement of forms and it is used in histopathology to describe measurements made from two dimensional sections. We used morphometry to assess the area and diameter of fat globules in biopsies with steatosis in $\mathrm{H} \& \mathrm{E}$ stained sections.

Steatosis was assessed in two areas: the pericentral area (zone III) and the periportal area (zone I). A special computer program was used to assess the area of parenchyma that contained steatosis. This computer program allowed the measurement to be done in two phases. At least 10 fields were chosen for morphometric analysis around portal tract areas, central vein areas, or nodules in cirrhotic livers. The mean diameter of fat globules was obtained (all measurements are in micrometers).

\section{DEFINITIONS}

Fat globules were graded quantitatively as follows.

(1) Microvesicular steatosis (microglobules): the mean diameter of fat globules plus one standard deviation is less than $15 \mu \mathrm{m}(\mathrm{M}+\mathrm{SD}$ $<15 \mu \mathrm{m}$ ).

(2) Macrovesicular steatosis (macroglobules): the mean diameter of fat globules minus one $\mathrm{SD}$ is equal to or larger than $15 \mu \mathrm{m}$ (M $-\mathrm{SD} \geq 15 \mu \mathrm{m})$.

(3) Mixed macrovesicular and microvesicular steatosis: the mean diameter of fat globules plus or minus one SD is more or less than $15 \mu \mathrm{m}$ $(\mathrm{M}+\mathrm{SD}>15 \mu \mathrm{m}$ or $\mathrm{M}-\mathrm{SD}<15 \mu \mathrm{m})$.

Parenchyma is defined by the area occupied by hepatocytes, Kupffer cells, and sinusoids without fatty changes.

Pleomorphism of fat globules is the degree of variation in the size of fat globules. This was obtained from the SD of the mean values of fat globules.

The distribution of fat globules was defined as periportal or pericentral when globules are confined to zone 3 or zone 1, respectively. Pan steatosis was defined as steatosis involving all zones or all nodules. Other forms of distribution of steatosis were defined by the presence of steatosis in part of the cirrhotic nodules or both pericentral and periportal liver cell parenchyma.

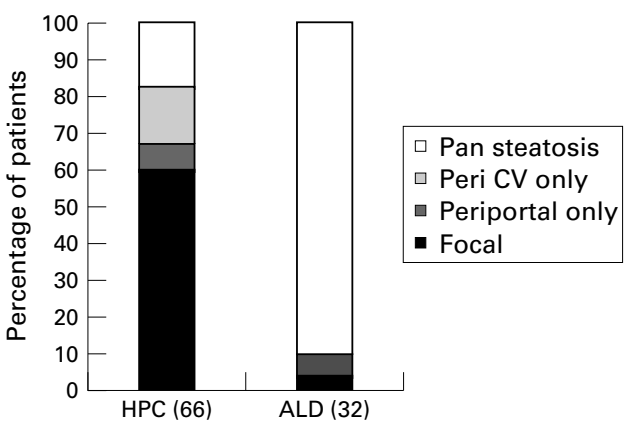

Figure 1 The patterns of distribution of fat globules in patients with chronic hepatitis $C$ (HPC) and alcoholic liver disease (ALD). CV, central vein. 


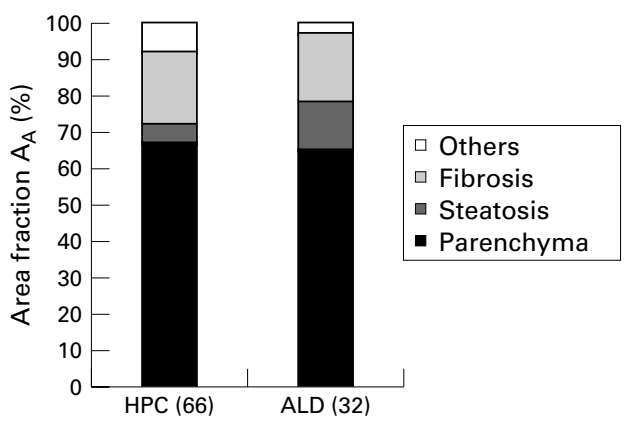

Figure 2 The area fraction values of fibrosis, steatosis, and other normal structures in patients with chronic hepatitis $C$ (HPC) and alcoholic liver disease (ALD).

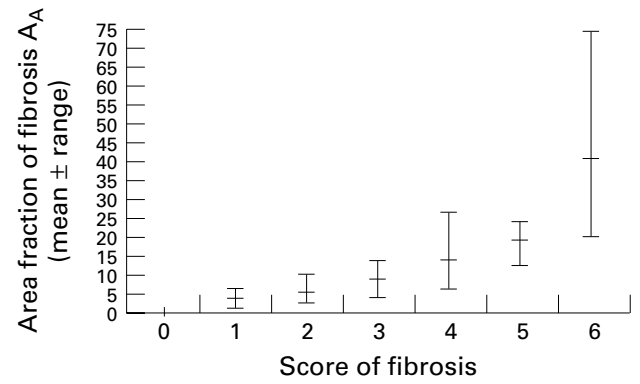

Figure 3 The area fraction of total fibrosis, including fibrosis in the portal tract area, in 86 patients with chronic hepatitis $C$ in relation to the Ishak modified score.

STATISTICAL ANALYSIS

Data were analysed using a software program EP1 INFO Version 6. The ANOVA test was used to derive the $\mathrm{p}$ value for normally distributed data. When Bartlett's test showed the variances in the samples to differ, the nonparametric Kruskal-Wallis $\mathrm{H}$ (equivalent to $\chi^{2}$ ) was used to obtain the $p$ value. Significance was set at $\mathrm{p}<0.05$ (two tailed).

The intraobserver reproducibility for assessing fibrosis and steatosis by stereological and morphometric techniques was tested. The coefficient of variation (CV) of fibrosis was $1.6 \%$ and the $\mathrm{CV}$ of steatosis was $3.8 \%$. Reproducibility is equal to $100-\mathrm{CV}$. The stereological and morphometric analyses were undertaken by a single observer (AMZ).

Table 1 The area fraction of fibrosis in relation to topography in liver biopsies from patients with chronic hepatitis $C$ (HPC) and chronic liver disease (ALD)

\begin{tabular}{lccl}
\hline Location of fibrosis & HPC $(n=86)$ & ALD $(n=32)$ & p Value \\
\hline Periportal & $9.04(6.37)$ & $3.59(3.16)$ & 0.000004 \\
Pericentral & $0.23(0.58)$ & $1.09(0.93)$ & 0.0045 \\
Pericellular & $2.23(4.81)$ & $3.91(2.73)$ & 0.044 \\
Septal and bridging & $8.41(5.12)$ & $7.63(10.24)$ & NS \\
Total & $19.14(10.59)$ & $15.97(12.51)$ & 0.008 \\
\hline
\end{tabular}

Results are expressed as mean (SD).

Table 2 Morphometric features of fat globules in patients with hepatitis $C$ (HPC) and alcoholic liver disease (ALD)

\begin{tabular}{lccl}
\hline & $H P C(n=68)$ & $A L D(n=32)$ & $p$ Value \\
\hline Diameter of fat globules $(\mu \mathrm{m})$ & $14.44(3.45)$ & $18.41(3.32)$ & 0.00002 \\
Pleomorphisim of fat globules $(\mu \mathrm{m})$ & $1.97(0.94)$ & $3.09(1.31)$ & 0.009 \\
\hline
\end{tabular}

Results are expressed as mean (SD).

\section{Results}

MORPHOLOGICAL CHANGES

Figure 1 shows the patterns of distribution of fat globules in patients with chronic hepatitis C and ALD. Pan steatosis was higher in ALD than in chronic hepatitis C. Periportal and other forms of distribution of fat were seen more frequently in patients with chronic hepatitis $\mathrm{C}$ than in those with ALD.

\section{STEREOLOGICAL CHANGES}

Figure 2 shows the $A_{A}$ values of fibrosis, steatosis, parenchyma, and other structures (portal tract areas, central vein areas combined) in patients with chronic hepatitis C and ALD. There was a significant difference $(p=0.008)$ in the $\mathrm{A}_{\mathrm{A}}$ of fibrosis between the two groups. The $A_{A}$ of steatosis was significantly higher in patients with ALD than in those with hepatitis C $(p=0.0002)$. There was no significant difference in the $\mathrm{A}_{\mathrm{A}}$ of parenchyma between the two groups. Figure 3 shows the stereological findings of fibrosis in patients with chronic hepatitis $\mathrm{C}$ in relation to the Ishak modified score of fibrosis. The area fraction of fibrosis increased exponentially from score 1 (portal fibrosis) to precirrhosis (score 5) to complete cirrhosis (score 6). In patients with cirrhosis the total percentage of fibrosis varied between $20 \%$ and $74 \%$ (approximately fourfold). Table 1 shows the percentage value of fibrosis in all topographical locations in chronic hepatitis C and ALD. In chronic hepatitis $\mathrm{C}$, periportal fibrosis was significantly higher than in the ALD group ( $p=0.00004)$. In ALD, pericentral fibrosis was significantly higher than in patients with chronic hepatitis C $(\mathrm{p}=0.0045)$.

\section{MORPHOMETRIC CHANGES}

Table 2 shows the mean diameter of fat globules in patients with chronic hepatitis C and ALD. The diameter of fat globules was significantly higher in patients with ALD than in those with chronic hepatitis C $(p=0.00002)$. Pleomorphism of fat globules was significantly higher in patients with ALD than in those with chronic hepatitis C $(\mathrm{p}=0.009)$.

Figure 4 shows the grades of fat globules in chronic hepatitis C and ALD. The proportion of macroglobules was higher in patients with ALD than in those with chronic hepatitis C $(p=0.0003)$. The proportion of microglobules was higher in chronic hepatitis C than in ALD $(\mathrm{p}=0.0003)$.

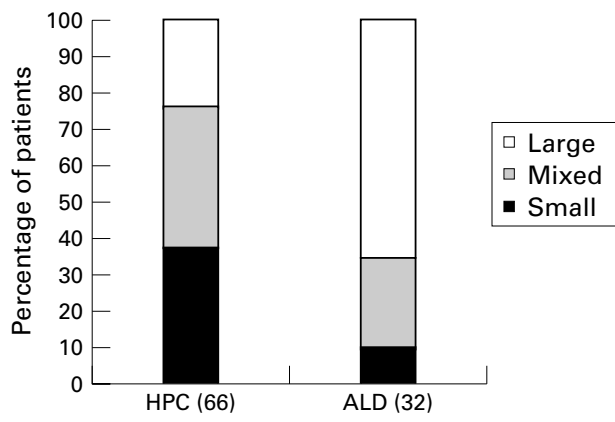

Figure 4 The grades of fat globules in chronic hepatitis $C$ (HPC) and alcoholic liver disease (ALD). 


\section{Discussion}

The primary aim of our study was to assess fibrosis and steatosis by quantitative methods in liver biopsy specimens from patients with chronic hepatitis C. Quantification of fibrosis by image analysis has been considered to be the gold standard of morphological measurement because its $\mathrm{CV}$ is very low, ${ }^{418}$ and values have been shown to correlate well with chemical $\alpha 2$ macroglobulin determination. ${ }^{16}$ Some authors have used morphometric techniques to assess fibrosis in patients with chronic hepatitis C. ${ }^{28}{ }^{29}$ We have shown in a previous study ${ }^{24}$ that stereology is superior to morphometry for quantifying fibrosis in ALD and NAFLD. In our current study, we have used stereology to assess fibrosis in patients with chronic hepatitis C. Stereological analysis has shown a significant increase in the area fraction with the increase in severity of chronic liver disease.

The modified Ishak scoring system ${ }^{19}$ has been used by pathologists as a semiquantitative estimation of fibrosis. Other systems have also been introduced to assess fibrosis. ${ }^{18}{ }^{30}$ However, these systems remain subjective. In addition, it may be difficult to compare the results between different studies using different scoring systems. The potential interobserver and intraobserver variation of grading and staging of fibrosis is very high. Our stereological technique for assessing fibrosis has shown a linear increase in the mean value of fibrosis in comparison with the modified Ishak score. However, there was an approximately fourfold variation (20-74\%) in the proportion of fibrosis within the same group of patients with established cirrhosis (score 6). A recent study has shown a similar fivefold difference within the same group of Knodell's score. ${ }^{30}$ This throws doubt on the validity of semiquantitative staging ${ }^{19}$ in the evaluation of new treatment strategies in hepatic fibrosis. Paradis et al have shown that necro inflammatory lesions and collagen content are important features for predicting the progression of liver disease in patients with chronic hepatitis C. ${ }^{31}$ Other authors have shown that serum collagen and fibrosis may lead to resistance to treatment with interferon in patients with chronic hepatitis C. ${ }^{32-34}$

The second goal of our work was to compare the degree of fibrosis at different locations in the patients with chronic hepatitis $\mathrm{C}$ and those with ALD. We found significant differences in the amount of fibrosis in relation to topography (in all locations) in patients with chronic hepatitis C and ALD. Periportal fibrosis was significantly higher in patients with chronic hepatitis C, whereas fibrosis was significantly higher in pericellular and pericentral vein areas in ALD. Several studies ${ }^{414}$ have shown that pericentral fibrosis lesions in ALD are the first apparent lesions in the sequence of events leading to alcoholic cirrhosis. The assessment of fibrosis in individual locations and the total areas yield a useful clue to the origin of fibrosis in both chronic hepatitis $\mathrm{C}$ and alcoholic disease. The significant difference in the location of fibrosis indicates that the origin of fibrosis differs between chronic hepatitis and ALD. It seems that the origin of fibrosis is predominantly pericentral and pericellular in ALD and predominantly periportal in chronic hepatitis C. Some authors have shown that pericentral fibrosis is a precursor lesion of cirrhosis. ${ }^{35} 36$ The natural history of liver fibrosis progression in patients with chronic hepatitis $\mathrm{C}$ has been studied. ${ }^{37}$ It has been estimated that, without treatment, $33 \%$ of patients have an expected median time to cirrhosis of less than 20 years. ${ }^{37}$

Steatosis of the liver is not a disease entity but a histological feature that may have clinical associations. It is the most common morphological feature seen in patients with ALD and some patients with chronic hepatitis C. We described a quantitative method to assess steatosis in all histopathological stages of liver disease in both groups of patients. Semigrading of steatosis has been described by many authors. ${ }^{7138}$ Semiquantitative and fully automated methods have also been described, ${ }^{38}$ but require special staining for fat. In semiautomated interactive methods, H\&E stained sections prepared for routine diagnosis can be used. In a previous study ${ }^{26}$ we assessed steatosis by two quantitative methods. Other authors have also used quantitative techniques to assess steatosis in liver biopsies. ${ }^{39}$ In our present study, both stereology and morphometry were used for assessing steatosis. Stereology has shown significant variation in $\mathrm{A}_{\mathrm{A}}$ of steatosis between patients with chronic hepatitis $\mathrm{C}$ and those with ALD. The diameter of fat globules differed significantly between the two groups. The highest degree of variation in the diameter of fat globules was seen in patients with ALD. In humans, fat globules initially formed in zone 3 coalesce to form large droplets. This may explain the variation in the diameter of fat globules in patients with ALD. Our results confirm the morphological findings that steatosis is focally distributed and is seen more frequently in zone 1 in patients with chronic hepatitis C than in those with ALD.

In a previous study, ${ }^{26}$ we found a good correlation between the morphological grading of fat globules and morphometric grades. Our present study has shown another application of the quantitative grades in patients with chronic hepatitis C. This system can be used easily to study the changes in steatosis when evaluating a new treatment strategy and also for follow up of patients with chronic hepatitis C.

In conclusion, stereology and morphometry are simple and reliable quantitative techniques for the measurement of fibrosis and steatosis in patients with chronic hepatitis C. Stereology offers a means of assessing fibrosis and steatosis in the entire biopsy specimen, whereas morphometry is very useful in assessing steatosis in relation to the size and diameter of fat globules. In view of the high reproducibility in intrafield assessment and low intraobserver variability, we recommend this method as one of the most important tools in the evaluation of new treatment strategies in hepatic fibrosis and for follow up of patients with chronic hepatitis C and ALD. 
1 Desmet VJ, Gerber M, Hoofnagle JH, et al. Classification of chronic hepatitis: diagnosis, grading and staging. Hepatolchronic hepatitis: diag
ogy 1994;19:1513-20.

2 Kage M, Shimamatu K, Nakashima E, et al. Long-term evolution of fibrosis from chronic hepatitis to cirrhosis in patients with hepatitis C: morphometric analysis of repeated biopsies. Hepatology 1997;25:1028-31.

3 Yano $M$, Kumada H, Kage $M$, et al. The long-term pathological evolution of chronic hepatitis C. Hepatology 1996;23:1334-9.

4 Caulet S, Fabre M, Schoevaert D, et al. Quantitative study cirrhosis. Virchows Archiv A Pathol Anat Histopathol 1989;416:11-17.

5 Chedid A, Mendenhall CL, Gartside P, et al and the VA Co-operative Study Group. Prognostic factors in alcoholic liver disease. Am 7 Gastroenterol 1991;86:210-16.

6 Baptista A, Bianchi L, De Groote J, et al (international Baptista A, Bianchi L, De Groote J, et al (international
group). Alcoholic liver disease: morphological group). Alcoholic liver disease: morphological manifestations:

7 Adler M, Schaffner F. Fatty liver hepatitis and cirrhosis in obese patients. Am f Med 1979;67:811-16.

8 Diehl AM, Goodman Z, Ishak KG. Alcohol-like liver disease in nonalcoholics: a clinical and histological comparison with alcohol-induced liver injury. Gastroenterology 1988;95:1056-62.

9 Klain J, Fraser D, Goldstein J, et al. Liver histology abnormalities in the morbidly obese. Hepatology $1989 ; 10$ 873-6.

10 Schmid M, Flury R, Buhler H, et al. Chronic viral hepatitis $\mathrm{B}$ and $\mathrm{C}$ : an argument against the conventional classification of chronic hepatitis. Virchows Archiv 1994;425:2218.

11 Lefkowitch JH, Schiff ER, Davis GL, et al. Hepatitis interventional therapy group. Pathological diagnosis of chronic hepatitis C: a multicenter comparative study with chronic hepatitis C. Gastroenterology 1993;104:595-603.

12 Czaja AJ, Carpenter HA. Sensitivity, specificity and predictability of biopsy interpretations in chronic hepatitis. Gastroenterology 1993;105:1824-32.

13 Uchimura, Y, Sata M, Kage M, et al. A histopathological study of alcoholics with chronic HCV infection: comparison with chronic hepatitis $\mathrm{C}$ and alcoholic liver disease. Liver 1995;15:300-6.

14 Nagore N, Scheuer PJ. The pathology of diabetic hepatitis. f Pathol 1988;156:155-60.

15 Gressner AM. Liver fibrosis: perspectives in pathobiochemical research and clinical outlook. Eur 7 Clin Chem Clin Biochem 1997;29:293-311.

16 Naveau S, Poynard T, Benattar C, et al. Alpha-2macroglobulin and hepatic fibrosis: diagnostic interest. Dis Dis Sci 1994;39:2426-32.

17 Yabu K, Kiyosawa K, Mori H, et al. Serum collagen type IV for the assessment of fibrosis and resistance to interferon therapy in chronic hepatitis C. Scand $\mathcal{f}$ Gastroenterol 1994; 29:474-9.

18 Chevallier M, Guerret S, Chossegros P, et al. A histological semiquantitative scoring system for evaluation of hepatic
fibrosis in needle liver biopsy specimen; comparison with fibrosis in needle liver biopsy specimen; compariso

19 Ishak K, Baptista A, Bianchi L, et al. Histological grading and staging of chronic hepatitis. F Hepatol 1995;22:696-9

20 Knodell RG, Ishak KG, Black WC, et al. Formulation and application of a numerical scoring system for assessing histological activity in asymptomatic chronic active hepatitis. Hepatology 1981;1:431-5.
21 Ryoo JW, Buschmann RJ. Morphometry of liver parenchyma in needle biopsy specimens from patients with alcoholic liver disease: preliminary variables for the diagno ; $2: 382-9$

22 Aronson DC, De Haan J, James J, et al. Quantitative aspects of the parenchyma-stroma relationship in experimentally induced cholestasis. Liver 1988;8:116-26.

23 James J, Bosch KS, Aronson DC, et al. Sirius red histophotometry and spectrophotometry of sections in the assessment of the collagen content of liver tissue and its application in growing rat liver. Liver 1990;10:1-5.

24 Zaitoun AM, Mardini H, Record CO. Quantitative assessment of fibrosis in liver biopsies from patients with alcoholic and non alcoholic liver disease. $\mathcal{f}$ Cell Pathol 1999;4:11-19.

25 Arnould L, Cosyn JP, Rondelez L, et al. Automated morphometric estimation of liver steatosis. Microsc Acta Suppl 1979;3:197-203.

26 Zaitoun AM, Mardini H, Record CO. Stereology and morphometry of steatosis In human alcoholic (ALD) and non alcoholic liver disease (NALD). Acta Stereologica 1998;17: 49-58.

27 Eaton S, Zaitoun AM, Record CO, et al. $\beta$-Oxidation in human alcoholic and non alcoholic hepatic steatosis. Clin Sci 1996;90:307-13.

28 Camma C, Giunta M, Linea C, et al. The effect of interferon on the liver in chronic hepatitis $\mathrm{C}$ : a quantitative evaluation of histology by meta-analysis. F Hepatol 1997;26:1187-99.

29 Lin X-Z, Horng M-H, Sun Y-N, et al. Computer morphometry for quantitative measurement of liver fibrosis. Comparison with Knodell's score, colorimetry and conventional description report. 7 Gastroenterol Hepatol 1998;13:75-80.

30 Bedoss P, Poynard T. The Metavir co-operative study group: an algoritham for grading the activity in chronic hepatitis C. Hepatology 1996;24;289-93.

31 Paradis V, Mathurin P, Laurent A, et al. Histological features predictive of liver fibrosis in chronic hepatitis $\mathrm{C}$ infection. $\mathcal{F}$ Clin Pathol 1996;49:998-1004.

32 Kim SR, Hayashi Y, Yoon S, et al. Prediction of efficacy of interferon treatment of chronic hepatitis C by multivariate analysis and a new classification. Pathol Int 1998;48:21520.

33 Hakozaki Y, Shirahama T, Katou M, et al. Long-term prognosis of chronic hepatitis $\mathrm{C}$ after treatment with interferon alpha $2 \mathrm{~b}$ and characterisation of incomplete responders. Am 7 Gastroenterol 1996;91:2144-9.

34 Yabu K, Kiyosawa K, Mori H, et al. Serum collagen type IV for the assessment of fibrosis and resistance to interferon therapy in chronic hepatitis C. Scand f Gastroenterol 1994; 29:474-9.

35 Worner TM, Lieber CS. Perivenular fibrosis in alcoholic liver injury: ultrastructure and histologic progression. Gastroenterology 1982;83:777-85.

36 Worner TM, Lieber CS. Perivenular fibrosis as precursor lesion of cirrhosis. FAMA $1985 ; 254: 627-30$

37 Poynard T, Bedossa P, Opolon P, for the OBSVIRC, METAVIR, CLINIVIR, and DOSIVIRC groups. Natural history of liver fibrosis progression in patients with chronic hepatitis C. Lancet 1997;349:825-32.

38 Auger J, Schoevaert D, Martin ED. Comparative study of automated morphometric and semiquantative estimations of alcoholic steatosis. Anal Quant Cytol Histol 1986;8:5662

39 De Craemer D, Pauwells M, Van Den Branden C. Alterations of peroxisomes in steatosis of the human liver: a quantitative study. Hepatology 1997;22:744-52. 